\title{
Cardiac magnetic resonance imaging demonstrates biatrial stunning after catheter ablation of persistent atrial fibrillation
}

\author{
Kai Muellerleile ${ }^{1 *}$, Michael Groth², Daniel Steven ${ }^{1}$, Boris Hoffmann', Jakob Lueker ${ }^{1}$, Arian Sultan ${ }^{1}$, Ulf K Radunski ${ }^{1}$, \\ Gunnar Lund ${ }^{2}$, Gerhard Adam², Thomas Rostock', Stephan Willems ${ }^{1}$
}

From 15th Annual SCMR Scientific Sessions

Orlando, FL, USA. 2-5 February 2012

\section{Summary}

This study evaluated the course of active left atrial (LA), LA appendage (LAA) and right atrial (RA) emptying with respect to ablation-related inflammation after persistent AF ablation by cardiac magnetic resonance imaging (CMR).

\section{Background}

Catheter ablation of persistent atrial fibrillation (AF) aims at reestablishing active atrial emptying. However, little is known on recovery of active atrial emptying and inflammation after successful ablation of persistent AF.

\section{Methods}

CMR was performed in 19 consecutive patients at baseline (BL) within 24 hours and at follow-up (FU) $5 \pm 2$ months after restoration of sinus rhythm by catheter ablation of persistent AF. Velocity encoded (VENC) MRI was used to assess active LA, LAA and RA emptying: Active LA and RA emptying were quantified by calculating the active emptying fraction (AEF) from transmitral VENC-MRI flow profiles. LAA function was quantified by measurements of peak a-wave velocities from VENC-MRI flow profiles perpendicular to the LAA orifice. Peri-atrial inflammation was assessed using edema-sensitive black-blood T2-weighted MRI.

\section{Results}

There was a significant increase in LA-AEF from 17 (12$26) \%$ at baseline to $25(23-36) \%$ at follow-up $(p<0.01)$. A significant increase was also found for LAA a-wave velocities from $45(30-67) \mathrm{cm} / \mathrm{s}$ at baseline to 62 (47-72) $\mathrm{cm} / \mathrm{s}$ at follow-up $(\mathrm{p}<0.01)$. Furthermore, RA-AEF significantly improved from $32(18-36) \%$ at baseline to 40 $(37-50) \%$ at follow-up $(\mathrm{p}<0.0001)$. Conversely, the area of peri-atrial edema on T2-weighted MRI decreased from 2340 (1944-2745) $\mathrm{mm}^{2}$ at baseline to 423 (237823) $\mathrm{mm}^{2}$ at follow-up $(\mathrm{p}<0.0001)$.

\section{Conclusions}

Catheter ablation of persistent AF results in inflammation-related biatrial stunning. Disappearance of inflammation is associated with recovery of active LA, LAA and RA emptying.

\section{Funding}

No external funding.

\section{Author details}

${ }^{1}$ Center for Cardiology and Cardiovascular Surgery, University Heart Center Hamburg, Hamburg, Germany. ${ }^{2}$ Department of Diagnostic and Interventional Radiology, University Medical Center Hamburg-Eppendorf, Hamburg, Germany.

Published: 1 February 2012

doi:10.1186/1532-429X-14-S1-P203

Cite this article as: Muellerleile et al:: Cardiac magnetic resonance imaging demonstrates biatrial stunning after catheter ablation of persistent atrial fibrillation. Journal of Cardiovascular Magnetic Resonance 2012 14(Suppl 1):P203. 